\title{
GENDER DIFFERENCES IN LIMB AND JOINT STIFFNESS DURING THE FEMCING LUNGE
}

\author{
Joanthan Sinclair, ${ }^{1, A, B, C, D}$ Lindsay Bottoms ${ }^{2, A, B, C, D}$ \\ ${ }^{1}$ Division of Sport Exercise and Nutritional Sciences, University of Central Lancashire, United Kingdom \\ ${ }^{2}$ School of Health, Sport and Bioscience, University of East London, United Kingdom

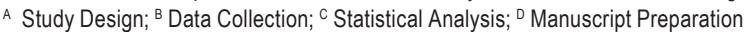 \\ Address for corpespondence: \\ Jonathan Sinclair \\ Division of Sport, Exercise and Nutritional Sciences \\ School of Sport Tourism and Outdoors \\ University of Central Lancashire, Preston Lancashire PR1 2HE, United Kingdom \\ E-mail: jksinclair@uclan.ac.uk
}

\begin{abstract}
Ahstract. The aim of the current investigation was to examine gender differences in limb and joint stiffness characteristics during the fencing lunge. Ten male and ten female fencers completed simulated lunge movements. Lower limb kinematics were collected using an eight camera optoelectric motion capture system which operated at $250 \mathrm{~Hz}$. Measures of limb and joint stiffness were calculated as a function of limb length and joint moments divided by the extent of limb and joint excursion. Gender differences in limb joint stiffness parameters were examined statistically using independent samples t-tests. The results showed firstly that both limb (male $=64.22$ \pm 19.12 , female $=75.09 \pm 22.15 \mathrm{~N} . \mathrm{kg} . \mathrm{m}$ ) and hip stiffness (male $=10.50 \pm 6.00$, female $=25.89 \pm 15.01 \mathrm{Nm} . \mathrm{kg} . \mathrm{rad}$ ) were significantly greater in female fencers. In addition it was also demonstrated that knee moment (male $=1.64 \pm 0.23$, female $=2.00 \pm 0.75 \mathrm{Nm} . \mathrm{kg}$ ) was significantly larger in females. On the basis of these observations, the findings from the current investigation may provide further insight into the aetiology of the distinct injury patterns observed between genders in relation to fencing.
\end{abstract}

Key WOrlls: fencing, biomechanics, limb stiffness

\section{Introduction}

Epee fencing is a recognised Olympic discipline during which the athletes are required to make contact with their opponent with their sword (Sinclair et al. 2010). Clinical research investigating the prevalence of injury in both elite and recreational fencers has demonstrated that injuries and pain connected specifically to training/ competition were apparent in $92.8 \%$ of all fencers (Harmer 2008). Importantly it was also shown that a high proportion of all injuries were experienced by the lower extremities (Harmer 2008). The repetitive high impact dynamic motions associated with fencing training and competition are considered to expose the lower extremity musculoskeletal structures to high levels of strain (Sinclair et al. 2010; Greenhalgh et al. 2013; Sinclair and Bottoms 2014). The lunge 
movement in particular which is the foundation of the majority of offensive fencing motions repeatedly exposes fencers to potentially detrimental impact forces (Sinclair et al. 2010).

In clinical biomechanics literature the importance of lower limb stiffness is now becoming acknowledged (Butler et al. 2003), as researchers and clinicians attempt to achieve more knowledge of how the musculoskeletal system responds to applied loads and attain additional insight into the aetiology of chronic lower limb injuries. Limb stiffness calculated as a function of the vertical force that is applied to a body divided by the resultant deformation of the limb as a function of the applied load (McMahon and Cheng 1990). During dynamic movements the contact limb is represented using a spring mass system (Latash and Zatsiorsky 1993), during which the contact limb is symptomatic of a linear spring and the mass of the athletes body is representative of the overall point mass (McMahon and Cheng 1990). Clinically, higher limb stiffness has been linked to an increased risk from bone-related injuries whereas insufficient limb stiffness has also been linked to soft tissue injury (McMahon et al. 2012).

Fencing is undertaken by both male and female athletes, previous analyses have examined gender differences in the mechanics of the fencing lunge. Sinclair and Bottoms (2013) investigated the kinetics and lower body kinematics of the lunge movement as a function of gender. It was demonstrated that females exhibited significantly greater knee abduction and hip adduction of the lead limb. Sinclair and Bottoms (2015) examined differences between genders in patellofemoral forces between male and female fencers who performed the lunge movement. Their results showed that female fencers were associated with significantly greater patellofemoral kinetics than males. Sinclair and Bottoms (2014) explored gender differences in the load experienced by the Achilles tendon, it was demonstrated that males were associated with a significantly larger Achilles tendon loads than female fencers.

However, gender differences in limb and joint stiffness parameters during the fencing lunge have not yet been explored in biomechanical literature. Therefore the aim of the current investigation was to examine gender differences in limb and joint stiffness characteristics as a function of the lunge movement.

\section{Methods}

\section{Participants}

Ten male and ten female epee fencers volunteered to take part in the current investigation. All were injury free at the time of data collection and provided written informed consent in accordance with the declaration of Helsinki. Participants were active competitive epee fencers who engaged in training a minimum of 3 sessions per week and were all right handed. The mean characteristics of the participants were males; age $27.22 \pm 4.08$ years, height $1.75 \pm 0.05 \mathrm{~m}$ and mass $74.31 \pm 6.05 \mathrm{~kg}$ and females; age $24.88 \pm 5.87$ years, height $1.67 \pm 0.07 \mathrm{~m}$ and mass 63.52 $\pm 4.66 \mathrm{~kg}$. The procedure was approved by the University of Central Lancashire ethics committee.

\section{Procedure}

Participants completed 10 lunges during which they were required to hit a dummy with their weapon and then return to a starting point which was determined by each fencer prior to the commencement of data capture. This allowed the lunge distance to be maintained. The fencers were also required to contact a force platform (Kistler Instruments Ltd., Alton, Hampshire) embedded into the floor (Altrosports $6 \mathrm{~mm}$, Altro Ltd,) of the biomechanics laboratory with their right (lead) foot. The force platform sampled at $1000 \mathrm{~Hz}$. 
Kinematic information was obtained using an eight camera optoelectric motion capture system (Qualisys Medical AB, Goteburg, Sweden) using a capture frequency of $250 \mathrm{~Hz}$. The current investigation utilized the calibrated anatomical systems technique (CAST) to quantify kinematic information (Cappozzo et al. 1995). To define the anatomical frame of pelvis, thigh, shank and foot retroreflective markers were positioned unilaterally to the medial and lateral malleoli, medial and lateral epicondyle of the femur and greater trochanter. Rigid technical tracking clusters were positioned on the shank and thigh segments. The tracking clusters comprised four retroreflective markers mounted to a thin sheath of lightweight carbon fibre with length to width ratios in accordance with Cappozzo et al. (1997). Static trials were obtained with participants in the anatomical position in order for the positions of the anatomical markers to be referenced in relation to the tracking clusters, following which markers not required for tracking were removed.

\section{Data processing}

Retroreflective marker positions were identified using Qualisys Track Manager in and then exported as C3D files to Visual 3D (C-Motion, Germantown, MD, USA) for further analysis. Ground reaction force and retroreflective marker trajectories were filtered at 50 and $12 \mathrm{~Hz}$ using a low pass Butterworth 4th order zero-lag filter (Sinclair, 2014). Hip, knee and ankle joint kinematics were calculated using an $X Y Z$ sequence of rotations (where $X$ represents sagittal plane; $Y$ represents coronal plane and $Z$ represents transverse plane rotations) (Sinclair et al. 2013). Newton-Euler inverse-dynamics were also adopted which allowed knee and ankle joint moments to be calculated. Kinetic/ kinematic measures from the hip, knee and ankle extracted for statistical analysis were 1) joint angular excursion (representing the angular displacement from footstrike to peak angle) and 2) peak joint moment.

Limb stiffness was quantified using a mathematical spring-mass model (Blickhan 1989). Limb stiffness was calculated from the ratio of the peak vertical GRF to the compression of the limb spring. Limb compression was calculated as the change in thigh length from footstrike to minimum thigh length during the stance phase (Farley and Morgenroth 1999). The torsional stiffness of the hip, knee and ankle joints were obtained as a product of the ratio of the change in joint moment to joint angular excursion (Farley and Morgenroth 1999).

\section{Statistical analyses}

Means and standard deviations were calculated as a function of gender for each outcome measure. Gender differences in limb and joint stiffness parameters were examined using independent samples t-tests with significance accepted at the $p \leq 0.05$ level. Effect sizes for all significant observations were calculated using partial eta ${ }^{2}\left(p^{2}\right)$. All statistical procedures were conducted using SPSS v22.0 (IBM SPSS, Inc., Chicago, IL, USA).

\section{Results}

Table 1 presents the gender differences in limb and joint stiffness. The results indicate that limb and hip joint stiffness parameters were significantly influenced as a function of gender.

\section{Joint kinetics and kinematics}

The results show that peak hip moment was significantly greater $\left(t_{(18)}=3.27, p<0.05, p \eta^{2}=0.33\right)$ in female fencers in comparison to males (Table 1). The results also indicate that hip excursion was significantly larger 
$\left(t_{(18)}=3.05, p<0.05, p \eta^{2}=0.27\right)$ in male fencers (Table 1). Finally, peak knee moment was shown to be significantly $\left(t_{(18)}=3.11, p<0.05, p \eta^{2}=0.28\right)$ greater in female fencers in comparison to males (Table 1).

Table 1. Limb and joint stiffness parameters as a function of gender

\begin{tabular}{lrrrrr} 
& \multicolumn{3}{c}{ Male } & \multicolumn{3}{c}{ Female } & \\
\cline { 2 - 6 } & mean & \multicolumn{1}{c}{ SD } & mean & \multicolumn{1}{c}{ SD } & \\
\hline GRF (N.kg) & 18.15 & 6.56 & 18.41 & 7.02 & \\
Limb compression (m) & 0.30 & 0.07 & 0.28 & 0.09 & $*$ \\
Limb stiffness (N.kg.m) & 64.22 & 19.12 & 75.09 & 22.15 & $*$ \\
Hip excursion (rad) & 0.32 & 0.20 & 0.27 & 0.19 & $*$ \\
Hip moment (Nm.kg) & 2.43 & 0.89 & 3.05 & 1.46 & $*$ \\
Hip stiffness (Nm.kg.rad) & 10.50 & 6.00 & 25.89 & 15.01 & $*$ \\
Knee excursion (rad) & 0.42 & 0.15 & 0.51 & 0.11 & \\
Knee moment (Nm.kg) & 1.64 & 0.23 & 2.00 & 0.75 & $*$ \\
Knee stiffness (Nm.kg.rad) & 4.58 & 2.54 & 4.24 & 2.22 & \\
Ankle excursion (rad) & 0.18 & 0.08 & 0.21 & 0.13 & \\
Ankle moment (Nm.kg) & 0.97 & 0.33 & 1.25 & 0.55 & \\
Ankle stiffness (Nm.kg.rad) & 7.88 & 6.70 & 7.93 & 4.35 & \\
\hline
\end{tabular}

* significant difference.

\section{Spring mass parameters}

Limb compression was found to be significantly larger $\left(t_{(18)}=3.14, p<0.05, p \eta^{2}=0.29\right)$ in male fencers in relation to females (Table 1). Limb stiffness was therefore shown to be significantly larger $\left(t_{(18)}=3.22, p<0.05\right.$, $p \eta^{2}=0.31$ ) in female fencers (Table 1). In addition hip stiffness was shown to be significantly larger in female fencers (Table 1).

\section{Discussion}

The current investigation aimed to determine whether there are gender differences in limb and joint when performing the lunge movement in fencing. To the authors' knowledge this represents the first study to examine gender differences in limb stiffness parameters in fencing.

The first key observation from the current investigation is that female fencers were associated with increased limb stiffness in relation to males. This finding does not agree with those of Granata et al. (2001) who showed that females were associated with reduced limb stiffness in relation to males when performing a hopping task. It is proposed that this difference relates to the different functional demands of hopping tasks in comparison to the fencing lunge (Sinclair and Bottoms 2013). This finding relates principally to the significant increases in limb compression that were observed in females as the peak vertical GRF magnitude did not differ significantly between genders. This observation may have relevance clinically as increased levels of limb stiffness, such as those observed in female runners, have been linked to the aetiology of bony injuries (McMahon et al. 2012). In addition to this, the findings from this study also confirmed that hip joint stiffness was significantly larger in female fencers. This observation relates to the significant reduction in hip excursion and corresponding increase in hip moment noted in females. Given the similar joint stiffness parameters observed between genders at the knee and ankle joints at appears that hip stiffness is the key contributor to the differences in limb stiffness. 
A number of studies have examined the relationship between limb stiffness and athletic performance (Bret et al. 2002; Farley and Gonzalez 1996; Heise and Martin 1998; Hobara et al. 2010). The extent of limb stiffness has been shown to be related to the utilization of the stretch-shortening cycle in dynamic movements (Brughelli and Cronin 2008). During the eccentric phase enhanced limb stiffness as a function of a reduction in limb compliance allows for maximum return of the stored energy during the concentric phase (Latash and Zatsiorsky 1993). This suggests that female fencers may be able to make more effective use of the stretch shorten cycle than males, indicating that they may be able to recover their position and progress onto the next attack more efficiently. Therefore whilst the increased limb stiffness observed in female may place them at increased risk from lower limb injury, it may also promote a corresponding increase in movement efficiency around the piste.

The increase in peak sagittal knee moment may also provide insight into the distinct injury patterns in females. Female athletes are at much greater risk of developing patellofemoral pain than age matched males (Wilson 2007). This finding concurs with the observations of Sinclair and Bottoms (2015), who demonstrated that both knee moment and patellofemoral loads were greater in female fencers. The knee joint moment profiles from the current study indicate that the load at the knee is larger in female fencers. Therefore, this finding re-enforces the conclusions from Sinclair and Bottoms (2015) as the consensus regarding the aetiology of patellofemoral pain is that symptoms are the function of excessive knee joint loading (Fulkerson and Arendt 2000).

In conclusion, although gender differences the mechanics of the fencing lunge have been examined extensively, the current knowledge regarding the effects of gender on limb and joint stiffness parameters is limited. The present investigation therefore adds to the current knowledge by providing a comprehensive comparative evaluation of the limb and joint stiffness characteristics of male and female fencers. On the basis that hip/ limb stiffness and knee moment were shown to be significantly greater in female fencers, the findings from the current investigation may provide further insight into the aetiology of the distinct injury patterns that have been noted between male and female athletes. Clinically the outcomes from the current investigation indicate that female fencers may be more susceptible to overuse injuries than males.

\section{Acknowledgements}

Our thanks go to Glen Crook for his technical assistance.

\section{References}

Blickhan R. The spring mass model for running and hopping. J Biomech. 1989; 22: 1217-1227.

Butler R.J., Crowell H.P., Davis I.M. Lower extremity stiffness: implication for performance and injury. Clin Biomech. 2003; 18: 511-517.

Bret C., Rahmani A., Dufour A.B., Messonnier L., Lacour J.R. Leg strength and stiffness as ability factors in $100 \mathrm{~m}$ sprint running. J Sports Med Phys Fitness. 2002; 42: 274-281.

Brughelli M., Cronin J. A review of research on the mechanical stiffness in running and jumping: methodology and implications. Scand J Med Sci Sports. 2008; 18: 417-426.

Cappozzo A., Catani F., Leardini A., Benedet M.G., Della C.U. Position and orientation in space of bones during movement: Anatomical frame definition and determination. Clin Biomech. 1995; 10: 171-178.

Cappozzo A., Cappello A., Croce U., Pensalfini F. Surface-marker cluster design criteria for 3-D bone movement reconstruction. IEEE Trans Biomed Eng. 1997; 44: 1165-1174.

Farley C.T., Gonzalez O. Leg stiffness and stride frequency in human running. J Biomech. 1996; 29: 181-186.

Farley C.T., Morgenroth D.C. Leg stiffness primarily depends on ankle stiffness during human hopping. J Biomech. 1999; 32: $267-273$.

Fulkerson J.P., Arendt E.A. Anterior knee pain in females. Clin Ortho Rel Res. 2000; 372: 69-73. 
Granata K.P., Padua D.A., Wilson S.E. Gender differences in active musculoskeletal stiffness. Part II. Quantification of leg stiffness during functional hopping tasks. J Electromyogr Kines. 2001; 12: 127-135.

Greenhalgh A., Bottoms L., Sinclair J. Influence of surface on impact shock experienced during the fencing lunge. J App Biomech. 2013; 29: 463-467.

Harmer P.A. Getting to the point: injury patterns and medical care in competitive fencing. Current Sports Medicine Reports. 2008; 7 : 303-307.

Heise G.D., Martin P.E. Leg spring characteristics and the aerobic demand of running. Med Sci Sports Exerc. 1998; 30: $750-754$.

Hobara H., Inoue K., Gomi K., Sakamoto M., Muraoka T., Iso S., Kanosue K. Continuous change in spring-mass characteristics during a 400 m sprint. J Sci Med Sport. 2010; 13: 256-261.

Latash M.L., Zatsiorsky V.M. Joint stiffness: Myth or reality? Hum Mov Sci. 1993; 12: 653-692.

McMahon T.A., Cheng G.C. The mechanics of running: how does stiffness couple with speed? J Biomech. 1990; $23: 65-78$.

McMahon J.J., Comfort P., Pearson S. Lower limb stiffness: considerations for female athletes. Strength Cond J. 2012; 34: 70-73.

Sinclair J., Bottoms L., Taylor K., Greenhalgh A. Tibial shock measured during the fencing lunge, the influence of footwear. Sp Biomech. 2010; 9: 65-71

Sinclair J., Bottoms L. Gender differences in the kinetics and lower extremity kinematics of the fencing lunge. Int J Perform Anal Sport. 2013; 13: 440-451.

Sinclair J., Taylor P.J., Bottoms L. The Appropriateness of the Helical Axis Technique and Six Available Cardan Sequences for the Representation of 3-D Lead Leg Kinematics During the Fencing Lunge. J Human kinet. 2013; 37: 7-15.

Sinclair J. Effects of barefoot and barefoot inspired footwear on knee and ankle loading during running. Clin Biomech. 2014; 29: 395-399.

Sinclair J., Bottoms L. Gender differences in the Achilles tendon load during the fencing lunge. Balt J Health Phys Activity. 2014; 6: 199-204.

Sinclair J., Bottoms L. Gender Differences in Patellofemoral Load during the Epee Fencing Lunge. Res Sports Med. 2015; 23: 51-58.

Wilson T. The measurement of patellar alignment in patellofemoral pain syndrome: are we confusing assumptions with evidence? J Orthop Sports Phys Ther. 2007; 37: 330-341.

Cite this anticle aS: Sinclair J., Bottoms L. Gender Differences in Limb and Joint Stiffness during the Fencing Lunge. Central European Journal of Sport Sciences and Medicine. 2015; 11 (3): 39-44. 